\title{
Adénome prostatique, hormones et androgénothérapie
}

\author{
Pierre COSTA \\ Service d'Urologie-Andrologie, C.H.U. de Nîmes
}

\section{RÉSUMÉ}

La prostate est androgèno-dépendante. L'adénome prostatique ou hyperplasie bénigne de la prostate (HBP) a une prévalence histologique extrêmement élevée mais tous les hommes porteurs d'une hyperplasie bénigne de la prostate histologique n'en souffrent pas. L'âge et la présence d'androgènes circulants sont les déterminants les plus importants de l'apparition d'une HBP. Toutefois, les taux d'androgènes plasmatiques des hommes porteurs d'une HBP ne sont pas différents de la population générale. La suppression des androgènes provoque une diminution du volume de la prostate. L'administration d'androgènes exogènes est suivie d'une re-croissance prostatique. Mais lors des études sur la substitution androgénique des hommes hypogonadiques, le volume de la prostate n'augmente pas ou peu et semble normal sous traitement. La revue de la littérature permet de penser que l'adénome prostatique ne représente pas une contre-indication à la supplémentation androgénique de l'homme présentant un déficit partiel au total en androgènes. Toutefois la plupart des études ont exclu les hommes porteurs d'une HBP, et une étude spécifique devrait être entreprise.

Mots clés : Hyperplasie bénigne de la prostate, andropause, traitement androgénique substitutif.

\section{INTRODUCTION}

La prostate est androgéno-dépendante. Sa croissance normale nécessite la présence d'androgènes circulants. L'hormone mâle active au sein du tissu prostatique est la dihydrotestostérone (DHT) obtenue par conversion, au sein du tissu prostatique, de la testostérone ( $\mathrm{T}$ ) circulante par la 5 alpha-réductase. Les hommes atteints de pseudohermaphrodisme mâle, lié à un déficit en 5 alpha-réductase, n'ont pas de développement prostatique mais une prostate vestigiale [10].

L'adénome prostatique ou hyperplasie bénigne de la prostate (HBP) est une tumeur bénigne dont la prévalence histologique est extrêmement élevée. Les études autopsiques [4] ont montré la présence de signes histologiques d'HBP chez $50 \%$ des hommes arrivés dans leur cinquième décennie et que cette prévalence augmentait régulièrement avec l'âge pour atteindre $90 \%$ à 80 ans.

Tous les hommes porteurs d'une HBP histologique n'en souffrent pas. La prévalence des troubles urinaires liés à l'existence d'une obstruction cervico-urèthrale secondaire à l'HBP, ou HBP symptomatique, est en France de $8 \%$ de 50 à $59 \mathrm{ans}, 14 \%$ de 60 à 69 ans et de $27 \%$ de 70 à 79 ans [19].

L'âge et la présence d'androgènes circulants sont les deux plus importants déterminants de l'apparition d'une HBP chez l'homme [1], la race et l'ethnie semblant aujourd'hui moins importants. La castration précoce ou l'hypopituitarisme empêche le développement d'une $\operatorname{HBP}[11,15]$.

Aucune différence n'a été montrée entre le taux d'andro-

Correspondance : Professeur Pierre Costa, Service d'Urologie- Andrologie, C.H.U. de Nimes, Hôpital Gaston Doumergue, 5 Rue Hoche, 30029 Nimes Cédex 4. 
gènes plasmatiques des hommes porteurs d'une HBP et la population générale [14]. Le taux plasmatique de T diminue avec l'âge chez les hommes porteurs d'HBP comme dans la population générale. Certains auteurs ont rapporté un taux de DHT circulante plus élevé dans la population avec HBP $[9,18]$.

Le taux plamatique moyen d'oestrogènes (E) augmente avec l'âge mais aucune différence significative n'a été retrouvée en comparant des hommes porteurs d'HBP à d'autres [9].

Les hypothèses actuelles incriminent un déséquilibre relatif entre le taux circulant de testostérone, qui diminue avec l'âge, et celui d'oestrogènes, qui se maintient, en association avec une augmentation conséquente du taux plasmatique de la Sex Hormone Binding Globulin (SHBG) [6]. La présence de DHT est essentielle à la croissance des cellules prostatiques mais n'est pas directement responsable de leur prolifération. Celle-ci dépend de la présence de facteurs de croissance activateurs comme l'EGF, le KGF et l'IGFs [6], normalement équilibrés par des facteurs de croissance inhibiteurs comme le TGFß.

\section{PROSTATE ET VARIATION DES ANDROGÈNES CHEZ L'ADULTE}

La suppression des androgènes par une castration bilatérale chirurgicale ou traumatique, l'utilisation d'agonistes du LHRH ou d'anti-androgènes provoquent une diminution de volume de la prostate [5]. Cette diminution de volume, qui peut atteindre $30 \%$, est de $27 \%$ avec le finastéride (inhibiteur de la 5 alpha-réductase [21]). Parallèlement le taux de PSA total plasmatique diminue de 50\% après 6 mois de traitement et cette diminution est stable après 3 ans de traitement ; le risque de présenter une rétention aiguë d'urine diminue de $57 \%$ et celui d'être opéré de $34 \%$ [22].

L'administration d'androgènes exogènes ou la suppression des traitements réalisant une castration médicale est suivi par une re-croissance prostatique. Toutefois cette croissance s'arrête lorsque la prostate a atteint sa taille initiale et la réponse à l'androgène ne provoque pas d'hypertrophie prostatique [2].

\section{ANDROPAUSE, ANDROGÉNOTHÉRAPIE ET PROSTATE}

Toutes les études publiées sur la supplémentation androgènique des hommes hypogonadiques rapportent des résultats concordant $[3,7,12,17,20]$ :

- le volume prostatique n'augmente pas ou peu et semble normal sous traitement,

- il n'y a pas d'apparition de troubles mictionnels,

- le PSA augmente peu ou n'augmente pas. Des taux éle- vés pouvant faire évoquer l'apparition d'un cancer prostatique ne sont pas atteints.

\section{HBP et androgénothérapie}

Dans la plupart de ces études, les patients porteurs d'HBP sont exclus. Morley [17] situe l'incidence de l'HBP dans les populations d'hommes traités par androgènes à seulement $1 \%$.

De ce fait il n'est pas possible préciser, à partir de l'étude la littérature, l'impact éventuel de la supplémentation androgénique sur la prostate d'hommes présentant un déficit partiel ou total en androgènes. Néanmoins, toutes les données rapportées ci-dessus vont en faveur de l'absence de toxicité prostatique de l'androgénothérapie.

\section{Surveillance prostatique sous androgénothérapie}

Considérant que la prostate est une cible privilégiée des androgènes, la plupart des auteurs [13] recommandent une surveillance prostatique chez les patients supplémentés en androgènes. Cette surveillance sera basée sur :

- le toucher rectal (TR),

- le taux de PSA plasmatique,

- l'échographie prostatique endorectale.

La première visite de contrôle sera envisagée 3 mois après l'initiation du traitement puis les consultations se tiendront tous les 6 mois pendant 2 ans, puis tous les ans. Il n'est pas nécessaire d'envisager une biopsie prostatique pré-thérapeutique si TR, PSA et échographie sont normaux [16].

\section{Prostate, androgénothérapie et caractéristiques de l'androgène}

Un androgène non convertissable en DHT ou non aromatisable en E2 pourrait avoir moins d'impact sur la prostate [8]. Toutefois, un tel androgène serait moins intéressant pour la minéralisation et le système cardio-vasculaire.

\section{CONCLUSIONS ET RECOMMANDATIONS}

Cette revue de la littérature permet de penser que l'adénome prostatique ne peut pas représenter une contre-indication à la supplémentation androgénique de l'homme souffrant d'un déficit partiel ou total en androgène.

Toutefois les études analysées pêchent par le manque de recul et l'absence quasi constante de patients porteurs d'HBP dans les populations étudiées. Une étude spécifique chez les hommes porteurs d'une HBP est hautement recommandée, pour analyser :

- l'effet de l'androgénothérapie sur la prostate, 
- le risque carcinogénétique,

- la survenue de troubles mictionnels ou de complications obstructives comme une rétention urinaire,

- l'impact du traitement sur la qualité de vie liée à la sphère urinaire.

\section{RÉFÉRENCES}

1. BARRY M.J. et al. : Epidemiology and natural history of BPH. Committee 1. In : Cockett A.T.K., Khoury S., Aso Y., Chatelain C., Denis L., Griffithe K., Murphy G. eds. The 3rd International Consultation on BPH, 1995 : SCI, 1996.

2. BEHRE H.M. : Prostate volume in treated and untreated hypogonadal men in comparison to age-matched controls. Clin. Endocrinol., 1994, 40: 341-349.

3. BEHRE H.M., VON ECKARDSTEIN S., KLIESCH S. et al. : Long-term substitution therapy of hypogonadal men with transscrotal testosterone over 7-10 years. Clin. Endocrinol., 1999, $50: 629-635$.

4. BERRY S.J., COFFEY D.S, WALSH P.C., EWING L.L. : The development of human benign prostatic hyperplasia with age. J. Urol., 1984, 132: 474-479.

5. FRICK J., JUNGWIRTH A., ROVAN E. : Androgens and the prostate. In: Nieschlag E., Behre H.M. eds. Testosterone : action, deficiency, substitution. Berlin, Springer-Verlag, 1998 : 259- 291.

6. GRIFFITHS K. et al. : The regulation of prostatic growth. Committee 3. In : Cockett A.T.K., Khoury S., Aso Y., Chatelain C., Denis L., Griffithe K., Murphy G. eds. The 3rd International Consultation on BPH. 1995 : SCI 1996.

7. GOOREN L.J.G. : A ten-years safety study of the oral androgen testosterone undecanoate. J. Androl., 1994, 15 : 212-215.

8. GOOREN L.J.G. : Options of androgen treatment in the aging male. The Aging Male, 1999, 2 : 73-80.

9. HAMMOND G.L. : Serum steroids in normal males and patients with prostatic diseases. Clin. Endocrinol., 1978, $9:$ 113-121.

10. IMPERATO-MCGINLEY J, et al. : Steroid 5 alpha-teductase deficiency in man: an inherited form of male pseudohermaphrodism. Science, 1974, $186: 1213-1215$.

11. JIE-PING W. et al. : The prostate $41-65$ years post-castration. An analysis of 26 eunuchs. Chin. Med. J., 1987, 100:271-272.

12. KENNY A.M. et al. : Short-term effects of intramuscular and transdermal testosterone on bone turnover, prostate symptoms, cholesterol and hematocrit in men over age 70 with low testosterone levels. Endocrine Res., 26 : 153-168.

13. KIM C.Y. et al. : Endocrine and metabolic aspect including treatment. In Erectile dysfunction. 1st international consultation on erectile dysfunction, July 1-3, 1999, Paris. Jardin A., Wagner G., Khoury S., Giuliano F., Padma-Nathan H., Rosen R. eds. Plymonth, Health Publication Ltd, Plymbridge Distributors, $2000: 207-240$.

14. LAWSON R K. : Etiology of BPH, In : Lepor H., Lawson R.K. eds. Prostate Diseases. WB Saunders Company, 1993 : 89-95.

15. MOORE R.A. : Benign hypertrophy and carcinoma of the prostate. Occurence and experimental production in animals. Surgery, 1944, 16 : 152-167.

16. MORALES A. : Andropause, androgen therapy and prostate safety. The Aging male, 1999, $2: 81-86$.

17. MORLEY J.E. et al. : Androgen deficiency in aging men : role of testosterone replacement therapy. J. Lab. Clin. Med., 2000, 135 : 370-378.

18. PARTIN A.W. et al. : Influence of age and endocrine factors on the volume of BPH. J. Urol., 1991, $145:$ 405-409.

19. SAGNIER P.P. et al. : Impact of symptoms of prostatism on level of bother and quality of life of men in the french community. J. Urol., 1995, $153: 669-673$.
20. SNYDER P.J., PEACHEY H., BERLIN J.A. et al. : Effects of testosterone replacement in hypogonadal men. J. Clin. Endocrinol. Metab., 2000, $85: 2670-2677$.

21. STONER E. : The clinical effects of a 5 alpha-reductase inhibitor, finasteride, on BPH. The Finasteride study group. J. Urol., 1992, $147: 1298$ 1302.

22. STONER E. : Three years safety and efficacy data in the use of finasteride in the treatment of BPH. Urology, 1994, $43: 284-289$.

\author{
ABSTRACT
Benign prostatic hyperplasia, hormones and androgen therapy.

Pierre COSTA

The prostate is an androgen dependent organ. Benign prostatic hyperplasia (BPH) has a high prevalence in histological studies, but all the affected men do not present symptoms. Aging and the presence of androgens are the main determinants of BPH. However, androgen blood levels were not higher in patients with BPH than in the general population. Suppression of androgens induced a decrease in prostatic volume. Androgen replacement therapy resulted in a re-increase of prostatic volume, but during androgen replacement therapy the prostate volume was only slightly increased, if any, thus remaining within normal range.

The present review of the literature indicates that $\mathrm{BPH}$ is not a contra-indication for androgen replacement therapy in men with partial or complete androgen deficiency. However, it must be noticed that in most of the studies published so far subjects with BPH have been excluded. A specific study involving such patients is to be undertaken.

Key Words: Benign prostatic hyperplasia, aging, androgen replacement therapy. 